\title{
La responsabilidad social corporativa en el Perú. Realidad versus discurso
}

\author{
MIGUEL A. LEÓN UNTIVEROS*
}

Los peruanos se han vuelto [...] más y más achorados, aventados, bravazos, chongueros, conchudos, mecedores, moscas, recurseros, vivos y criollos [...].

Francisco Durand

SUMARIO: INTRODUCCIÓN.- I. LA RESPONSABILIDAD SOCIAL CORPORATIVA.- II. RESPONSABILIDAD SOCIAL CORPORATIVA VERSUS RENTABILIDAD.- III. CONCEPCIÓN DE RESPONSABILIDAD SOCIAL CORPORATIVA Y SU IMPACTO EN LA CONCEPCIÓN DE EMPRESA.- IV. RESPONSABILIDAD SOCIAL CORPORATIVA Y CORRUPCIÓN.- V. FRAGMENTACIÓN Y POSIBILIDAD. VI. CONCLUSIONES.

\section{INTRODUCCIÓN}

En nuestro país, la conciencia de la responsabilidad social corporativa (RSC), al menos a nivel del discurso, ocupa un espacio significativo. Por ello, resulta de suma importancia evaluar su puesta en práctica en atención a su realidad económica y social.

Así, en este trabajo presentamos el estado del arte junto con algunas críticas importantes sobre la RSC y su noción más amplia: la sostenibilidad corporativa (partes I al IV). Luego pasamos a contrastar el modelo discursivo con la realidad peruana para concluir que, tal y como están las cosas, el único modelo de responsabilidad social corporativa es el friedmaniano (rentabilidad), y explicamos las razones de esta conclusión (parte V).

\section{LA RESPONSABILIDAD SOCIAL CORPORATIVA}

\section{1. Responsabilidad social corporativa y filantropía}

Uno de los conceptos con los que se identifica a la RSC es la filantropía. Tal es el caso de Alemania ${ }^{1}$. Asimismo, se la puede concebir como cierto

* Es abogado por la Pontificia Universidad Católica del Perú y MBA por Maastricht School of Management, Holanda. Es profesor de la Facultad de Derecho de la Pontificia Universidad Católica del Perú y de Planeamiento Estratégico en la Universidad Inca Garcilaso de la Vega, además de socio del Estudio León \& Abogados Asociados, presidente del Centro de Investigación de las Asociaciones Público-Privadas - CIAPP y fundador del Centro de Investigación para el Mejoramiento del Servicio Legal - CIMES LEGAL. E-mail: miguel.leon@ pucp.edu.pe.

1 LEAL, Walter Filho y Paulina S. PAWLAK. «Federal Republic of Germany». En Samuel O. Idowu y Walter Leal Filho (editores). Global practices of corporate social responsibility. Heidelberg: Springer, 2009 , pp. 62 y siguientes. Sin embargo, no debe caerse en la tentación de simplificar los alcances de la filantropía. Ella se entiende como un tipo de compassionate love - que traducimos como «amor 
tipo de reciprocidad: tratar al otro como este nos trata, lo cual es de carácter voluntario y no en cumplimiento de un acuerdo ${ }^{2}$.

No obstante lo anterior, resulta interesante evaluar los motivos que dan lugar a la reciprocidad, en el sentido antes señalado ${ }^{3}$ :

a. Motivos de benevolencia

i. Benevolencia: compasión y predisposición

ii. Entrega a fin de obtener la satisfacción del otro

iii. Mostrar y probar simpatía por el otro

b. Motivos neutros

iv. Interés (por ejemplo, por razones de beneficios indirectos en favor del que da)

v. Deber y propiedad

vi. Ser generoso o aparentarlo

c. Entrega, inequidad y estatus

vii. Estatus: enfatizar la desigualdad entre las personas

viii. Poder y crédito moral del agente

ix. Jerarquía

x. Diferencias morales

Sin embargo, no cabe identificar la filantropía o la reciprocidad con la RSC. Como veremos, su concepto ha sufrido un desarrollo importante en términos de su alcance.

\section{I.2. Formulación de la RSC}

Es interesante revisar las diversas formulaciones matemáticas empleadas por la economía sobre la RSC, puesto que proporcionan una idea de sus inicios y nos permite apreciar las diferencias con su concepción actual.

La RSC fue inicialmente formulada por Milton Friedman, quien señaló lo siguiente ${ }^{4}$ :

$$
\text { RSC }=\text { Profit }
$$

De este modo, Friedman sostenía que la responsabilidad social de la empresa (RSE) consistía únicamente en el uso rentable de los recursos

compasivo»-, medido a través de las siguientes variables: empatía, asistencia social, capacidad de ayuda, voluntarismo y altruismo/caridad. FEHR, Beverley y Susan SPRECHER. «Compassionate love: conceptual, measurement, and relational issues». En Berverly Fehr, Susan Sprecher y Lynn G. Underwood (editoras). The science of compassionate love: theory, research, and applications. West Sussex: Blackwell Publishing, 2009, pp. 35 y siguientes.

2 Kolm, Serge-Christophe. Reciprocity. An economics of social relations. Cambridge: Cambridge University Press, 2008, p. 1.

3 Ibíd., pp. 56 y siguientes.

4 Friedman, Milton. Capitalism and freedom. Chicago: University of Chicago Press, 1962; y, del mismo autor, «The social responsibility of business to increase its profits». The New York Times Magazine, 13:33, septiembre de 1970, pp. 122-126. 
conducentes al incremento de la rentabilidad (ganancias) siguiendo las reglas del juego.

Posteriormente, John Elkington formuló lo siguiente"

$$
\mathrm{RSC}=\mathrm{ECV}+\mathrm{ECVL}+\mathrm{SOCV}
$$

LA RESPONSA-

BILIDAD SOCIAL

CORPORATIVA

EN EL PERÚ. REA-

LIDAD VERSUS

DISCURSO

$\mathrm{ECLV}=$ valor ecológico

SOCV $=$ valor social

Para Elkington, la empresa debía generar un valor económico (ser rentable), lo que debía engarzar con actividades favorables al ambiente natural para crear un valor social; es decir, debía realizar actividades en beneficio de la vida y de la comunidad. Esta concepción de RSC es más amplia que la friedmaniana. Entre uno y otro no hay contradicción sino una relación de conjunto/subconjunto (John Elkington/ Milton Friedman).

Fueron Carroll y Buchholtz ${ }^{6}$ quienes finalmente brindaron la formulación actual de la RSC:

$$
R S C=E R C+L G R+E T R+P H R
$$

Donde:

$\mathrm{ECR}=$ responsabilidad económica

LGR $=$ responsabilidad legal

$\mathrm{ETR}=$ responsabilidad ética

$\mathrm{PHR}=$ responsabilidad filantrópica

En consecuencia, para Carroll y Buchholtz la responsabilidad social corporativa es la conjunción de cuatro variables:

- Responsabilidad económica implica que la empresa sea rentable en sus operaciones económicas al cuidar que los precios de sus bienes y servicios sean justos.

- Responsabilidad legal implica que la empresa cumpla con las normas jurídicas.

5 Elkington, John. Cannibals with forks: the triple bottom line of 21st century business. Oxford: Capstone, 1997. Las siglas se mantienen en inglés.

6 CARROLL, A. B. y A. K. BUCHOLTZ. Business and society: ethics and stakeholder management. Quinta edición. Cincinnati: South Western Pub, 2003, pp. 33 y siguientes. 
- Responsabilidad ética implica que la empresa cumpla con aquellos deberes que no forman parte del Derecho objetivo pero que favorecen a los stakeholders.

- Responsabilidad filantrópica implica que la empresa, además de cumplir con sus deberes legales y éticos, voluntariamente participe en programas que favorezcan la comunidad y el medio ambiente.

Una vez más, debe observarse que entre este último concepto de RSC y los de Milton Friedman y John Elkington no existe contradicción sino una relación de conjunto y subconjunto.

Sin embargo, si solo se entiende que la nueva concepción de RSC es una sumatoria de sus variables, entonces cabe que, al aplicarla en un caso concreto, algunas de estas sean negativas o deficientes - por ejemplo, la responsabilidad ética一, mientras que las demás sean positivas o eficientes, de tal manera que el resultado total sea positivo o eficiente.

Por ello, junto a esta concepción cuantitativa de la RSC, debe añadirse su concepción cualitativa. De este modo, la RSC también debe formularse de la siguiente manera:

\section{$R S C=E R C^{\wedge} \mathrm{LGR}^{\wedge} \mathrm{ETR}^{\wedge} \mathrm{PHR}$}

Es decir, la responsabilidad social no solo es la suma de cada uno de los elementos antes señalados, sino que representa el cumplimiento satisfactorio de cada uno de ellos. De esta manera, basta el incumplimiento de uno solo para que la empresa no sea responsable?

Por otro lado, cabe formular la existencia de una serie de relaciones entre estas variables de la RSC. Muestra la manera en que sus variables interactúan entre sí, ya sea de forma positiva o de forma negativa. Piénsese en el caso de una norma jurídica injusta. ¿Qué debe primar: la responsabilidad legal o la ética? ${ }^{8}$ Como hemos explicado, esta dicotomía

7 Como se recordará, en el caso de una proposición compuesta conjuntiva, esta solo es verdadera si lo es cada uno de sus elementos conformantes. Véase por todos COPI, Irvin M. Introducción a la lógica. Néstor Míguez (traductor). Buenos Aires: Editorial Universitaria de Buenos Aires, 1962, pp. 219 y siguientes. Hedman, Shawn. A first course in logic. An introduction to model theory, proof theory, computability, and complexity. Oxford: Oxford University Press, 2006, p. 4.

8 Esta situación conlleva demasiadas cuestiones como para ser tratada adecuadamente en este trabajo. Nos limitamos a señalar lo siguiente: (i) el modelo analítico para esta situación es el sistémico y, de acuerdo con él, la relación entre las cuatro responsabilidades puede ser la de un reforzamiento positivo (círculo virtuoso) o la de un reforzamiento negativo (círculo vicioso); (ii) la relación sistémica antes señalada da lugar a diversos escenarios, y se muestra así que no debe entenderse como si se tratara de compartimentos estancos; (iii) por otro lado, la evaluación de la conducta empresarial a la luz del modelo sistémico de la RSC plantea la dicotomía ética frente a Derecho. Efectivamente, al estar frente a una norma injusta, el empresario estará frente al dilema ético-jurídico de cumplirla o no. $Y$ de no existir en el sistema jurídico algún órgano competente, básicamente el empresario tomará sus decisiones en función de dicha situación, es decir que optará por el cumplimiento de la norma injusta. Esta solución cambiaría radicalmente si hubiera alguna autoridad competente a la que la empresa pueda recurrir y si esta tuviera la competencia de liberarla del cumplimiento de la norma injusta en razón de una laguna axiológica. Sobre este concepto, véase ALCHOURRón, Carlos E. y Eugenio BuLYGIN. Introducción a la metodología de las ciencias jurídicas y sociales. Buenos Aires: Astrea, 1987, pp. 157 y siguientes. 
entre Derecho y moral no solo requiere de una adecuada configuración analítica, sino que el sistema jurídico debe ser capaz de resolver este tipo de conflictos, más aún cuando el fundamento último de la RSC son los derechos fundamentales de la persona?

\section{3. Iniciativas corporativas de responsabilidad social}

De acuerdo con Philip Kotler y Nancy Lee ${ }^{10}$, en cuanto a las iniciativas corporativas, existen seis opciones:

1. Promoción de causas sociales. La empresa provee recursos para una causa social.

2. Causas vinculadas con el marketing. La empresa se compromete a donar o a contribuir con un porcentaje de ventas de un determinado producto.

3. Marketing social corporativo. La empresa, de forma voluntaria, desarrolla y/o implementa alguna iniciativa para mejorar la salud pública, la seguridad, el medio ambiente o el bienestar social.

4. Filantropía corporativa. La empresa dona por caridad o por alguna otra causa social relevante.

5. Voluntariado. La empresa promueve en sus empleados, proveedores y demás miembros la prestación de su tiempo para causas sociales.

6. Prácticas de responsabilidad en los negocios. La empresa adopta y ejecuta prácticas comerciales e inversiones que promueven el bienestar y la protección del medio ambiente.

De acuerdo con los autores antes mencionados, la puesta en marcha de una o varias de estas iniciativas corporativas de responsabilidad social genera los siguientes efectos ${ }^{11}$ :

a. Incremento de ventas y de participación en el mercado objetivo

b. Fortalecimiento del posicionamiento de la marca

c. Mejoramiento de la imagen y de la estructura corporativa

d. Disminución de costos operativos

e. Mejoramiento del atractivo de inversionistas y analistas financieros

En consecuencia, de acuerdo con la literatura, la RSC no solo coadyuvaría al desarrollo sostenible, sino que también tendría sentido

9 Hemos de anotar que no se trata de la tutela directa, sino también indirecta, de un derecho fundamental. La cuestión vendría a ser planteada a nivel judicial por el titular de la RSC, sin perjuicio de que lo haga por su parte el titular del derecho. Sin embargo, lo interesante de este caso se presenta por el lado del titular de la RSC (la empresa), puesto que no es titular de una obligación jurídica.

10 KotLER, Philip y Nancy LEE. Corporate Social Responsability. Doing the most good for your company and your cause. Hoboken, Nueva Jersey: John Wiley \& Sons, 2005, pp. 23 y siguientes.

11 lbíd., pp. 10 y 11. 
empresarial $^{12}$, lo que define el principal ideal en la relación entre RSC y rentabilidad.

\section{RSC VERSUS RENTABILIDAD}

Si bien hemos señalado que la relación entre RSC y rentabilidad es ideal, ahora hemos de profundizar en los aspectos reales de esta relación. ¿Es cierto que ser éticamente responsable conduce a ser más rentable? ${ }^{13}$

Sobre esta cuestión, David Vogel ${ }^{14}$ señala que, si bien algunos estudios muestran que sí existe correlación positiva entre RSC y rentabilidad, otros muestran que dicha relación puede ser negativa, neutral o mixta.

Resulta difícil formular conclusiones generales sobre la relación entre RSC y rentabilidad, habida cuenta de que las investigaciones realizadas miden diferentes situaciones. No obstante, es posible que la RSC sea razonable y real para algunos negocios en determinadas circunstancias.

Sobre esto último, Vogel señala que, para un grupo de empresas, la RSC sí tiene sentido empresarial ${ }^{15}$. De un lado, para una primera subcategoría de estas empresas, la RSC es parte de sus planes estratégicos y de identidad corporativa. De otro, para una segunda subcategoría de empresas, la RSC tiene sentido en la medida en que estas se ven afectadas por la acción de activistas. Fuera de estas condiciones, las empresas no tendrían razón alguna para encaminar sus acciones hacia una RSC.

Por lo tanto, la RSC no guarda una relación directa con la rentabilidad, por lo que recién bajo dos tipos de condiciones las empresas contarían con incentivos reales para ser responsables socialmente.

\section{CONCEPCIÓN DE RSC Y SU IMPACTO EN LA CONCEPCIÓN DE EMPRESA}

Ingresamos ahora a una parte de la teoría de la responsabilidad social donde predomina una nueva terminología y donde no es fácil hacer distinciones correspondientes entre sí, lo que da lugar a su uso indiscriminado ${ }^{16}$. Resulta conveniente, así, desarrollar algunas precisiones.

\footnotetext{
12 Sin embargo, más adelante veremos que esta feliz conclusión no es real, tanto a nivel internacional como nacional.

13 Philipp Kotlker y Nancy Lee sostienen que existe una correlación positiva entre rentabilidad y RSC. KOTLER y LeE. Corporate Social Responsibility... Óp. cit., pp. 10 y 11.

14 Vogel, David. The market for virtue: the potential and limits of Corporate Social Responsibility. Washington: Brookings Institution Press, 2005, pp. 29 y siguientes. 


\section{III.1. La ciudadanía corporativa}

Este concepto se refiere al rol de las empresas en la sociedad e indica que deben prestar más atención a aspectos sociales y ambientales. Este fenómeno se ha llamado también «corporación civil» ${ }^{17}$.

\section{III.2. La sostenibilidad corporativa ${ }^{18}$}

Por «sostenibilidad corporativa» se entiende la satisfacción de las necesidades empresariales a la vez que se requiere el mantenimiento de recursos sociales, económicos y naturales. En ello no solo importa el empleo eficiente de dichos recursos, sino que también debe considerarse el impacto de las actividades económicas en la sociedad y en la naturaleza. Así, existen tres elementos constitutivos de la sostenibilidad corporativa: (i) la RSC, (ii) la teoría del stakeholder y (iii) la rendición de cuentas de la corporación ${ }^{19}$.

Esta concepción da lugar a la formulación matemática de la socio-eficiencia y de la eco-eficiencia:

$$
\text { Socio }-/ \text { Eco }- \text { eficiencia }=\frac{\sum \text { valor agregado }+ \text { externalidades positivas }}{\sum \begin{array}{c}
\text { impacto negativo acumulado sobre } \\
\text { la sociedad y la naturaleza }
\end{array}}
$$

LA RESPONSA-

BILIDAD SOCIAL CORPORATIVA

EN EL PERÚ. REALIDAD VERSUS DISCURSO

Así, la socio/eco-eficiencia es la relación entre los efectos positivos y negativos de la actividad empresarial sobre la sociedad.

\section{3. La respuesta social corporativa ${ }^{20}$}

También conocida como $\mathrm{RSC}_{2}$, por ella se entiende una orientación práctica de las actividades de la empresa. Su preocupación central no es la rectitud de las expectativas sociales, sino la búsqueda de vías para su adecuada implementación.

En el nivel micro, se trata de un área en el que interesa la implementación práctica de valores y cultura corporativos a través de estrategias. En el nivel macro, deben medirse los incentivos positivos y negativos para el logro de las metas públicas esperadas en las políticas económicas y sociales ${ }^{21}$. 


\section{4. El desempeño social corporativo 22}

Por este término se entiende el conjunto de indicadores concernientes a los resultados de la RSC. Este concepto requiere que una empresa no solo se mantenga saludable en términos financieros, sino que trata de que se disminuyan los costos sociales y ambientales ligados con su desempeño ${ }^{23}$. Su relación con la RSC es muy estrecha y la podemos caracterizar como de medio-fin (desempeño social corporativo-RSC).

\section{5. El gobierno corporativo}

De acuerdo con Keinert, «el gobierno corporativo comprende esencialmente un amplio rango de principios y mecanismos que aseguran el debido control del poder a fin de proteger los intereses de los stakeholders de la empresa $»^{24}$.

\section{III.6. La responsabilidad social corporativa: el enfoque basado en los derechos humanos}

Como es sabido, a lo largo de una serie de documentos los organismos internacionales hacen referencia a los derechos humanos entendidos como exigencias frente al Estado. Sin embargo, ello también puede ser relevante para el caso de las empresas: es decir, que sean las empresas igualmente llamadas a respetar los derechos humanos, con especial énfasis en las grandes multinacionales, a fin de contribuir al desarrollo sostenible ${ }^{25}$.

Una vez explicados expeditivamente los diversos conceptos vinculados con el rol social de las empresas, en el Gráfico 1 mostramos la relación entre los conceptos más destacados por la literatura.

Como puede verse, actualmente la RSC va quedando rezagada por la sostenibilidad corporativa, y se espera que en el futuro cercano dicho desplazamiento sea aun mayor.

\section{7. La nueva concepción de empresa: en enfoque sistémico}

A lo largo de lo señalado anteriormente, de forma implícita queda clara la decadencia de una concepción mecanicista de la empresa, o sea, aquella que la concibe como un fenómeno aislado de su entorno ${ }^{26}$.

Frente a esta primera concepción, existe en la actualidad la concepción holística de la empresa. Esta no la considera como una mera sumatoria

22 Traducimos de este modo el término corporate social performance.

23 KEINERT. Corporate Social Responsibility... Óp. cit., p. 45.

24 lbíd., p. 46.

25 Ibíd., pp. 75 y siguientes.

26 Desde este punto de vista mecanicista, es claro que la RSC no tendría sentido conceptual. Incluso se podría concebir a esta última como un concepto extravagante y atentatorio contra la naturaleza de la empresa. 


\section{Gráfico 1}

Ciudadanía corporativa, RSC y sostenibilidad corporativa y su relación con el desarrollo sostenible

Macro-nivel: economía
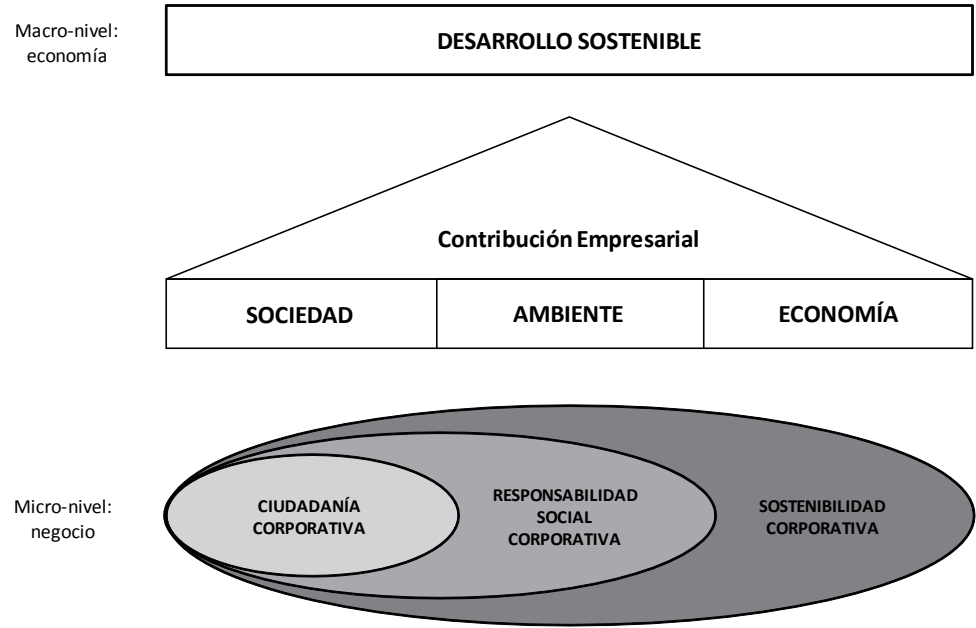

Fuente: Walter Filho Leal and Paulina S. Pawlak "Federal Republic of Germany". En: Global Practices of Corporate Social Responsibility. Samuel O. Idowu and Walter Leal Filho (Ed.). Heidelberg: Springer, 2009. p. 66.

de sus elementos, sino que ha de entenderse que estos se encuentran en una relación interactiva, lo que da lugar a sinergias y produce un valor agregado que no puede explicarse desde la perspectiva mecanicista.

Para Russell Ackoff, los elementos de la empresa son los siguientes:

1. organización democrática, en razón de que cada elemento afecta al sistema;

2. economía de mercado interno, donde los elementos del sistema realizan transacciones entre sí;

3. estructura organizacional multidimensional, que equivale a consumo interno, consumo externo y usuarios;

4. planificación interactiva; y

5. sistema de soporte de decisiones ${ }^{27}$.

De acuerdo con esta concepción, la categoría conformada por los stakeholders constituye un elemento esencial de la empresa y hace referencia a un individuo o grupo de individuos que son directamente afectados por el desempeño de la empresa en tanto que también dichos individuos 
pueden influir en el futuro de ella ${ }^{28}$. Solo desde esta concepción holística son plenamente justificables afirmaciones como esta: «La mayor función social de las empresas concebidas como un sistema social es crear y proveer trabajos productivos» ${ }^{29}$.

\section{8. Teoría del stakeholder}

En este punto es importante tener una idea más clara del rol central que ocupan los stakeholders en la concepción de la RSC. El siguiente gráfico muestra los componentes que son afectados - positiva y negativamente- por actividades empresariales.

\section{Gráfico 2}

\section{Mapa de los Stakeholders de la empresa}

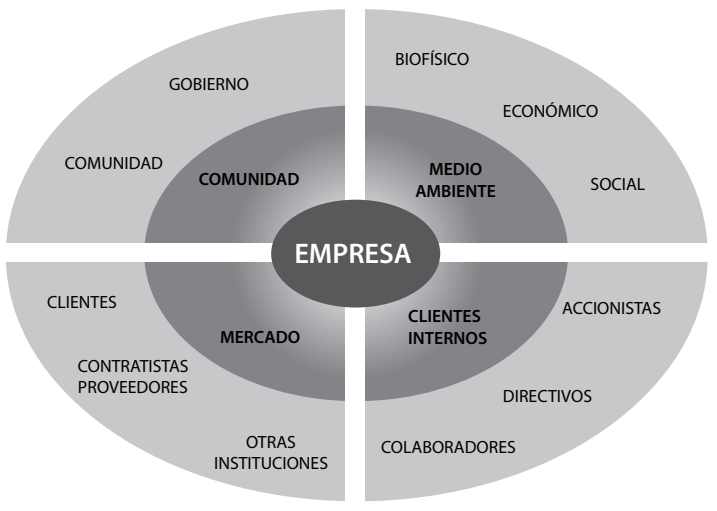

Una primera concepción del stakeholder es que se trata de «cualquier grupo de individuos quien [sic] puede ser afectado o es afectado por el logro de los objetivos de una organización». ${ }^{30}$

Asimismo, de acuerdo con Lynda Bourne, por definición el stakeholder cuenta con una participación (stake) en la actividad empresarial, y dicha participación puede provenir de cualquiera de las siguientes fuentes:

- Interés

- Derecho (legal o moral)

- Propiedad 
- Contribución en la formación del conocimiento o del recurso empresarial

En el cuadro que sigue, mostramos la descripción completa que Bourne hace sobre cada una de las categorías que dan lugar al stakeholder, lo cual es sumamente necesario con el fin de conocer la situación concreta de cada tipo de participación:

\section{Gráfico 3}

\section{Definición de los tipos de participación del stakeholder}

\section{LA RESPONSA- \\ BILIDAD SOCIAL CORPORATIVA \\ EN EL PERÚ. REA- LIDAD VERSUS DISCURSO}

\begin{tabular}{|c|c|}
\hline Interés & $\begin{array}{l}\text { Persona o grupo de personas que es afectada por una decisión } \\
\text { relativa a la actividad empresa o sus resultados: } \\
\text { - Efecto del cierre de las calles con ocasión e un evento } \\
\text { público. } \\
\text { - Colaboración para la creación de un parque natural en otra } \\
\text { región. }\end{array}$ \\
\hline Derecho & $\begin{array}{l}\text { Ser tratado de determinada manera o tener una protección } \\
\text { específica de algún derecho: } \\
\text { - Derecho legal: (i) salud y seguridad laborales, (ii) privacidad. } \\
\text { - Derecho moral: activistas de protección del ambiente. }\end{array}$ \\
\hline Propiedad & $\begin{array}{l}\text { Una circunstancia por la cual una persona o un grupo de ellas } \\
\text { tiene un título legal sobre un bien material o inmaterial: } \\
\text { - Propiedad intelectual. } \\
\text { - Acciones y/o participaciones en una sociedad. }\end{array}$ \\
\hline Conocimiento & $\begin{array}{l}\text { Conocimiento especializado o conocimiento organizacional } \\
\text { requerido para determinada actividad. }\end{array}$ \\
\hline $\begin{array}{l}\text { Impactoo } \\
\text { Influencia }\end{array}$ & $\begin{array}{l}\text { Los stakeholders pueden ser: } \\
\text { - Impactados por una actividad o sus resultados: personal, } \\
\text { consumidores, accionistas. } \\
\text { - Impactar o influir sobre la actividad o sus resultados: } \\
\text { patrocinadores, gobiernos (legislación, tributación), el público. }\end{array}$ \\
\hline Contribución & $\begin{array}{l}\text { Los stakeholders son respónsables por: } \\
\text { - El suministro de recursos: personal, material, financiamiento. } \\
\text { - Abogar por el éxito de los objetivos o las actividades, } \\
\text { intermediar entre las organizaciones y los equipos de trabajo, o } \\
\text { el desarrollo de las actividades empresariales. }\end{array}$ \\
\hline
\end{tabular}

Fuente: Linda Bourne. Stakeholder Relationship Management: a maturity model for organisational implementation. Farnham: Gower Publishing Limited y Ashgate Publishing Company, 2009. p. 66.

Como se observa, no hay homogeneidad entre stakeholders. Deberá entonces tenerse en cuenta el tipo de participación y/o de injerencia que se exige en concreto para determinar el contenido exacto de la RSC por cada caso. 


\section{RSC Y CORRUPCIÓN}

Un tema que resulta importante mencionar, al menos dentro de los límites de este trabajo, es la relación entre corrupción y empresa. Una cuestión principal es saber cómo es que se llega a ser una sociedad corrupta $^{31}$. Para Sharon Eicher ${ }^{32}$, la causa esencial radica en las instituciones débiles, tanto para la corrupción pública como para la privada. Esta situación (corrupción) permite que los agentes violen las instituciones sociales, con una mínima exposición a ser descubiertos y sancionados.

Por otro lado, las instituciones son débiles cuando están pobremente desarrolladas o cuando poseen escaso poder coercitivo. Ello da lugar a que las reglas sean ambiguas. En consecuencia, los líderes no están obligados a rendir cuentas de sus actos. Las prácticas de sobornos, nepotismo y otros tipos de inconductas son resultado de lo anterior.

En el caso del Perú, la situación nos resulta bastante obvia: la debilidad de las instituciones constituye la base de la presencia de la corrupción en ambos niveles (público y privado). De acuerdo con el Political Risk Service Group (2008), Excerpt from International Country Risk Guide, el Perú cuenta con un riesgo país de $63.5^{33}$. Asimismo, la variable de corrupción para el Perú es de 2.5 - para Estados Unidos de América esta es de 4 y para Venezuela es de 1, por ejemplo-.

Por consiguiente, resulta fácil entender la inconsistencia entre la RSC y la corrupción imperante en el Perú. Las empresas peruanas pueden sentirse extrañas - $y$ hasta inútiles - cuando se les exige responsabilidad social, mientras que la realidad obra en sentido opuesto.

\section{FRAGMENTACIÓN Y POSIBILIDAD}

Una cuestión importante sobre la RSC en el Perú es discutir su posibilidad no simplemente como discurso sino con miras a su implementación. Sobre ello, debemos explicar en primer lugar la situación económica del Perú.

\section{V.1. Estructura del mercado peruano.}

Francisco Durand ${ }^{34}$ postula que es necesario superar la dicotomía propuesta por José Matos Mar (Desborde popular y crisis del Estado) y

31 La cuestión, planteada de esta manera, nos permite postular lo siguiente: en una sociedad corrupta, ¿sus agentes -incluyendo las empresas- son corruptos? Si bien no es correcto generalizar, no debe perderse de vista el entorno en el que se desenvuelven los agentes - lo que, por cierto, determina en gran medida sus decisiones y el sentido de sus acciones-.

32 EICHER, Sharon. «Introduction: what corruption is and why it matters». En Sharon Eicher (editora). Corruption in international business. The challenge of cultural and legal diversity. Surrey y Burlington: Ashgate Publishing Limited and Gower Publishing Company, 2009, pp. 8 y siguientes.

33 Debe tenerse en cuenta que el Political Risk Service Group realiza este ranking sobre las siguientes variables: (i) estabilidad gubernativa; (ii) condiciones socioeconómica; (iii) perfiles de inversión; (iv) conflictividad interna; (v) conflictividad externa; (vi) corrupción; (vii) presencia político-militar; (viii) tensiones religiosas; (ix) Derecho y orden; (x) tensiones étnicas; (xi) responsabilidad democrática; y (xii) calidad de la burocracia.

34 DuRAND, Francisco. El Perú fragmentado. Lima: Fondo Editorial del Congreso del Perú, 2007, pp. 70 y siguientes. 
Hernando De Soto (El otro sendero) ${ }^{35}$ entre sector formal y sector informal para dar lugar a nuevas distinciones basadas en la presencia del Estado.

Por un lado, por rule of law se entiende el hecho de que, política y económicamente, existe un adecuado funcionamiento del mercado integrado con un verdadero Estado ${ }^{36}$. La situación opuesta es conocida como unrule of law, y en ella impera la violación de la norma, de la ley, de la Constitución y de las buenas costumbres ${ }^{37}$.

De este modo, Durand llega a proponer el siguiente espectro de legalidad y la ubicación de las distintas economías que operan en el mercado peruano:

\section{Gráfico 4}

Espectro de legalidad por sectores o economías

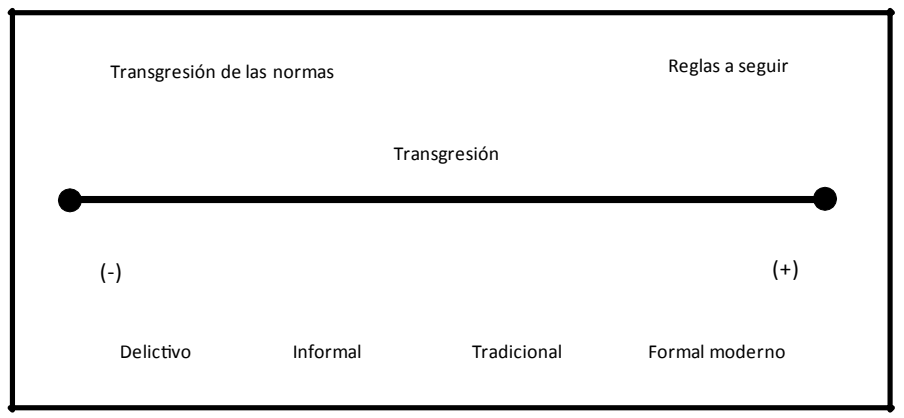

Fuente: Francisco Durand. El Perú Fragmentado. Lima: Fondo Editorial del Congreso del Perú, 2007. p. 41.

De acuerdo con el autor, ya no son dos sino cuatro los tipos de economía que actúan en el mercado peruano: (i) el formal-moderno; (ii) el tradicional; (iii) el informal; y (iv) el delictivo. Sin embargo, Durand emplea solo tres categorías y prescinde de la economía tradicional.

Por otro lado, no cabe concebir dichas economías como compartimentos estancos sino como capas transversales, presentes en todo el mercado peruano. Asimismo, la causa inmediata - pero no la única — de esta fragmentación es la debilidad o la ausencia del Estado peruano. Empero, no se piense que esta ausencia es homogénea en todo el país, pues se registran diversos grados o niveles de desinterés o de inexistencia de vías de comunicación entre los departamentos y Lima e incluso dentro de $\operatorname{Lima}^{38}$. mirada actual al trasporte público en Lima Metropolitana. Lima: CIES e IEP, 2009. En este trabajo, Bielich postula y demuestra que la gran ausencia del Estado es un factor determinante para la caótica situación del transporte público en la capital. Así, no ha de caerse en el simplismo de pensar que la presencia del Estado es homogénea en Lima Metropolitana, sino precisamente lo contrario. Ello dependerá, pues, del mercado en concreto.

LA RESPONSABILIDAD SOCIAL CORPORATIVA EN EL PERÚ. REALIDAD VERSUS DISCURSO 
Así, según Durand, las antiguas brechas que separan a los peruanos no han disminuido ni mucho menos se han eliminado, sino que han sido sustituidas por otras nuevas. Ello ha dado lugar a la coexistencia de varias economías dentro del país, tal y como se observa a continuación:

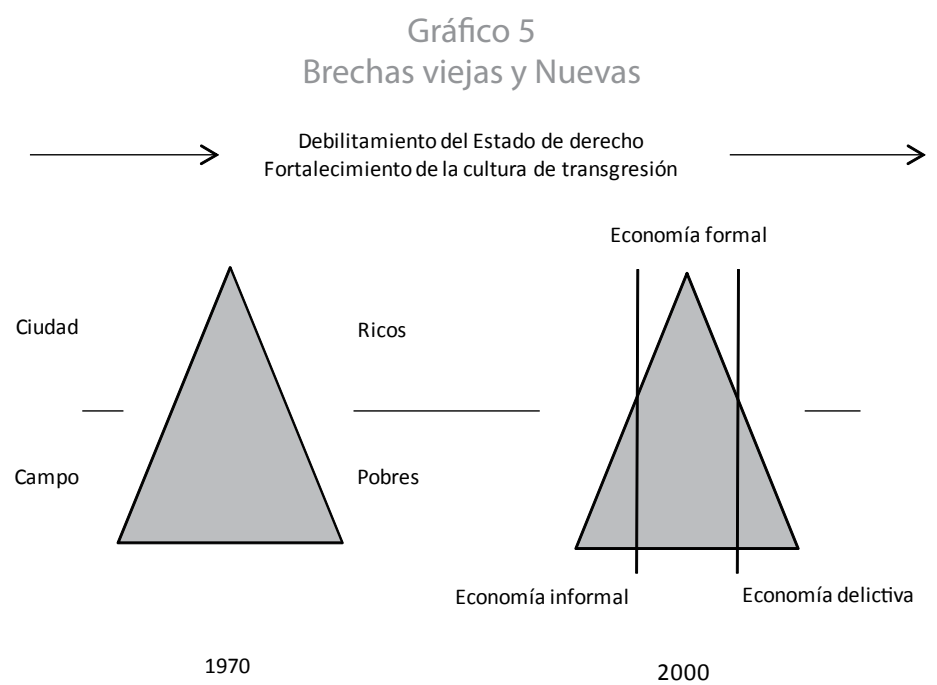

Fuente: Francisco Durand. El Perú Fragmentado: formalidad, informalidad y economía delictiva. Lima: Fondo Editorial del Congreso del Perú, 2007. p. 58.

Hagamos las siguientes observaciones sobre el gráfico anterior:

- Cuanto mayor es el debilitamiento del Estado de Derecho (unrule of law), más se fortalece la cultura de la transgresión.

- El actual debilitamiento del Estado es mucho mayor que el del pasado.

- En el pasado (hacia 1970), las antiguas brechas dieron lugar a dos tipos de economía, claramente diferenciados en términos geopolíticos: ciudad-ricos frente a campo-pobres.

- Actualmente (2000 en adelante), las antiguas brechas fueron sustituidas por nuevas, lo cual fragmentó aun más el mercado y dio lugar a tres economías: la formal, la informal y la delictiva.

- La pirámide social actual no tiene base — en el pasado sí la tenía-, pues no existe una relevante clase social media.

\section{V.2. Características y principios de la política peruana}

De acuerdo con Yusuke Murakami ${ }^{39}$, el Perú se presenta como una sociedad fragmentada cual «archipiélago»; es decir, dentro de la gran 
pirámide fragmentada que representa el país, existen múltiples pequeñas pirámides en su interior, sin vasos comunicantes entre sí. Al respecto, véase el siguiente gráfico:

\section{Gráfico 6}

Perú: sociedad fragmentada

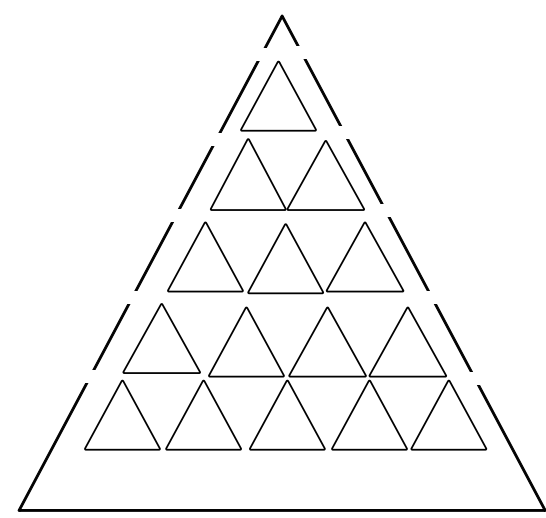

Fuente: Elaboración propia.
LA RESPONSA-

BILIDAD SOCIAL

CORPORATIVA

EN EL PERÚ. REALIDAD VERSUS

DISCURSO

Esta estructura social tiene sus bases en la época colonial y es una de las causas de que todavía no exista una identidad global peruana. Por otro lado, resulta sumamente interesante cuando Murakami señala que la política peruana se sustenta sobre dos principios ${ }^{40}$ :

a. Segmentación de la sociedad política peruana por relaciones autoritarias. Murakami, siguiendo a Julio Cotler, señala las siguientes características:

i. Relaciones de clientelismo con intereses individuales

ii. Surgimiento de la imagen del caudillo

iii. Verticalismo de estas relaciones

iv. Seguidores del grupo político que no participan en la toma de decisiones

v. Monopolización del poder, al punto de ser considerado «un derecho particular» $\mathrm{O}$ «una propiedad privada»

vi. Ausencia de colaboraciones a largo plazo entre los líderes y grupos políticos

b. Ausencia de líderes nacionales y del símbolo de integración. Murakami señala lo siguiente:

i. Un centro que se caracteriza con un «egoísmo frío» (Lima) 
ii. Inexistencia histórica de un plan de desarrollo de largo plazo

iii. Inexistencia de una clase media cuantiosa y cohesionada

iv. Falta de símbolos de cohesión del Perú como país

\section{3. ¿RSC en el Perú?}

Responderemos esta pregunta mediante el análisis de otras dos: ila presencia de las economías peruanas (formal, informal y delictiva) puede armonizar con el concepto actual de RSC? y, ila economía formal desarrolla sus actividades en atención a sus stakeholders?

La respuesta a la primera pregunta es clara si se tiene en cuenta que el Perú no está en un proceso de integración y reconducción de las economías informales y delictivas, sino que estas coexisten junto a la formal, la cual, a lo sumo, se ha limitado a tratar al ciudadano informal y delictivo como consumidor de los grandes supermercados, sin preocupación alguna sobre la procedencia de sus ingresos ${ }^{41}$. Esta acción, por lo demás, condice la existencia de nuevas brechas que sustituyen a las antiguas, conforme lo explicamos anteriormente.

Sin embargo, es todavía posible pensar que en el Perú existe un modelo de RSC, aunque ciertamente no el moderno. Efectivamente, como se ha señalado, la finalidad de las empresas formales se centra en la mejoría de sus rentas, lo cual se condice con el modelo friedmaniano de RSC.

Con respecto a la segunda pregunta, la respuesta es parcialmente afirmativa. La acción de la economía formal no incluye la totalidad de las categorías de los stakeholders, sino solo a los accionistas y/o «participacionistas» de empresas formales, con prescindencia de los demás stakeholders. Nuevamente, esta situación se condice con el modelo friedmaniano de RSC, en el que la responsabilidad social de la empresa únicamente consiste en el uso rentable de los recursos conducentes al incremento de la rentabilidad conforme con las reglas del juego.

Por otro lado, y como corolario de lo anterior, cabe indicar que la falta de consistencia entre la realidad peruana y la moderna RSC nos lleva a postular que la empresa en el Perú se concibe de modo mecanicista, y no holísticamente, habida cuenta, además, de que no es concebible, en nuestra economía, una relación positiva entre entorno (RSC) y empresa (rentabilidad).

\section{CONCLUSIONES}

1. La RSC no debe identificarse con la filantropía. Al margen de ello, debe tenerse encuentra que la filantropía es un tipo de amor 
compasivo, cuya motivación no siempre es encomiable, sino que puede ser neutra y hasta negativa.

1. Para Milton Friedman, la RSC se circunscribe a la rentabilidad. Este modelo fue superado por John Elkington, quien la formuló como una sumatoria de los siguientes valores: (i) económico, (ii) ecológico y (iii) social.

2. Carroll y Buchholtz postularon la forma actual de la RSC, presentada como la sumatoria de las responsabilidades económica, legal, ética y filantrópica.

3. Asimismo, la RSC no solo es la suma de cada uno de los elementos antes señalados, sino que es el cumplimiento satisfactorio de cada uno de ellos. De esta manera, basta el incumplimiento de uno solo para que la empresa no sea responsable.

4. Las variables de la RSC se interrelacionan en forma sistémica, lo cual nos permite explicar adecuadamente los conflictos entre el Derecho positivo y la ética. Como hemos señalado, además, esta dicotomía requiere que el sistema jurídico sea capaz de resolver este tipo de conflictos, más aún cuando el fundamento último de la RSC lo constituyen los derechos fundamentales de la persona.

5. Por otro lado, para una parte de la literatura sobre RSC, esta es per se provechosa para el empresario, puesto que tendría un impacto real sobre sus ganancias.

6. No obstante, David Vogel ha demostrado que dicha conclusión, aunque muy deseable, no es real, ya que la relación entre RSC y rentabilidad es contingente (positiva, negativa o neutra).

7. Por otro lado, junto con el concepto de la RSC existen otros tales como:

a. Ciudadanía corporativa

b. Sostenibilidad corporativa

c. Respuesta social corporativa

d. Desempeño social corporativo

e. Gobierno corporativo

8. Esta pluralidad de conceptos genera una gran confusión al definir qué se entiende por «empresa responsable».

9. A pesar de ello, la concepción actual de la RSC se basa en los derechos humanos, que deben ser respetados no solo por el Estado sino también por las empresas —en especial, por las multinacionales-.

10. Este nuevo concepto de RSC va ligado al concepto de desarrollo sostenible y da lugar a la sostenibilidad corporativa.

11. Asimismo, esta nueva concepción de responsabilidad corporativa exige una nueva concepción de empresa, que es facilitada precisamente por el enfoque sistémico.

\section{LA RESPONSA- BILIDAD SOCIAL CORPORATIVA EN EL PERÚ. REA- LIDAD VERSUS DISCURSO}


12. Según esta concepción, los stakeholders son un elemento esencial de la empresa.

13. No obstante la bondad del discurso actual sobre la RSC, existe una clara relación entre empresa y corrupción, sobre todo en países como el Perú, donde las instituciones son débiles.

14. Por otro lado, el mercado peruano no está lo suficientemente desarrollado como para adoptar el esquema moderno de RSC (Carroll y Buchholtz), habida cuenta de la coexistencia de tres economías (formal, informal y delictiva) que mantienen relaciones de conveniencia y que no constituyen otra cosa que nuevas brechas que profundizan la fragmentación del país (Durand).

15. Esta situación nos hace pensar que el único modelo posible de predicarse es el friedmaniano, según el que la responsabilidad social de la empresa únicamente consiste en el uso rentable de los recursos conducentes al incremento de la rentabilidad siguiendo las reglas del juego.

16. Finalmente, la inconsistencia entre la realidad peruana y la moderna RSC nos lleva a postular que la empresa en el Perú se concibe de forma mecanicista, y no holísticamente, habida cuenta, además, de que en nuestra sociedad no se concibe una relación positiva entre entorno (RSC) y empresa (rentabilidad). 